\title{
A LQR DESIGN WITH REJECTION OF DISTURBANCES AND ROBUSTNESS TO LOAD VARIATIONS APPLIED TO A BUCK CONVERTER
}

\author{
Luiz A. Maccari Jr. ${ }^{1}$, Rodolfo L. Valle ${ }^{2,3}$, André A. Ferreira ${ }^{2}$, Pedro G. Barbosa ${ }^{2}$, Vinícius F. Montagner ${ }^{1}$ \\ ${ }^{1}$ Universidade Federal de Santa Maria, Santa Maria - RS, Brasil \\ ${ }^{2}$ Universidade Federal de Juiz de Fora, Juiz de Fora - MG, Brasil \\ ${ }^{3}$ Centro Federal de Educação Tecnológica de Minas Gerais, Leopoldina - MG, Brasil \\ e-mails: luizmaccari@gmail.com,rodolfolacerda@leopoldina.cefetmg.br, andre.ferreira@ufjf.edu.br, \\ pedro.gomes@ufff.edu.br, vfmontagner@gmail.com
}

\begin{abstract}
This work presents a state feedback controller suitable to regulate the output voltage of DC-DC buck converters operating under input voltage disturbances and load variations. First, a state space model of the plant is given and the performance requirements are presented, in terms of bounds on rejection of disturbances, bounds on settling time of transient responses and robustness against load variations. Then, a control design procedure based on a linear quadratic regulator is proposed, having the following features: a) an $\mathscr{H}_{\infty}$ analysis condition to certify the bound on the rejection of disturbances, $b$ ) an eigenvalue evaluation, to ensure the bound on the settling times of the slowest mode in the transient responses, and $c$ ) a Lyapunov condition to ensure the robustness of the closed-loop system under load variations. The proposed design procedure produces control gains suitable for experimental implementation. For sake of comparison, a robust state feedback $\mathscr{H}_{\infty}$ controller with pole location constraints is given, and results in very large gains, which are not suitable for experimental implementation. Practical results with the proposed controller are shown, illustrating the very good performance of the system operating under input disturbance and load variations.
\end{abstract}

Keywords - Buck Converter, Linear Matrix Inequalities, Linear Quadratic Regulator, Robust $\mathscr{H}_{\infty}$ Control

\section{INTRODUCTION}

DC-DC converters have received increasing interest in several areas, as for instance computer energy sources, telecommunication equipment and sources of electrical vehicles. Robust control techniques that use fixed control gains to ensure stability and performance for DC-DC converters operating, for example, with variable load and also subject to disturbances are of great interest in industry [1], [2]. In general, a good trade-off between robustness and performance must be established, which demands knowledge of the plant to be controlled and of the controller to be used.

Among modern control techniques, based on optimization [3], the linear quadratic regulator (LQR) [4] deserves special attention, since it can guarantee good stability margins, establish suitable relationship between transient responses and

Manuscript received 26/04/2015; revised 09/10/2015; accepted for publication 08/12/2015, by recommendation of the Regular Section Editor Cassiano Rech. control signal energy, and also has the control gains easily computed by means of specialized computational packages. Application of LQR to power converters can be seen, for instance, using state observers, in [5]-[8]. A difficulty in application of LQRs is the choice of the cost function weighting matrices. To help this choice, genetic algorithms can be used, as in [9], [10].

The robust control of buck converters was addressed, for instance, in [11]-[13]. Linear matrix inequalities (LMIs) are interesting to deal with the design of controllers robust to parametric uncertainties and disturbances, as in [14], [15], where polytopic models of the plant were used to describe parametric uncertainties and LMIs were used to determine the gains of robust LQR controllers. LMIs were also used in [16]-[18] to provide robust controllers for boost converters. The control of DC-DC converters was also addressed using nonlinear techniques as, for instance, in [19]-[26].

These techniques were used with objective of dealing with specific problems, as saturation of the control signal [19], reduction of the start-up time [20] and rejection of load disturbances [11], as well as to ensure global stability for nonlinear converters. Despite providing good results, nonlinear techniques can be difficult in terms of control design or control implementation.

The contribution of this work is to provide a design procedure for an LQR controller applied to a buck converter, capable to ensure: (i) a predetermined rejection of disturbances on the input voltage, (ii) a predetermined settling time for the slowest mode in the transient responses and (iii) robustness to variations of the load in a predetermined interval. Property $(i)$ is ensured by means of the solution of the optimization problem based on the $\mathscr{H}_{\infty}$ norm [27], [28], property (ii) is ensured imposing a bound on the real part of the eigenvalues of the closed-loop system and property (iii) is certified by means of the existence of a Lyapunov function computed using LMIs.

As an example of application of the control for a DCDC buck converter subject to input voltage disturbances and to important load variations, one can cite a fully electrical vehicle. During the fast acceleration and deceleration of the vehicle, large current peaks can produce sudden variation of the battery voltage level. In this way, it is important to perform the conditioning of the energy supplied by the battery bank before it is used to feed the embedded circuits (as for instance, digital controllers, sensors, actuators, analog conditioning circuits, communication and lighting systems). These auxiliary circuits require supplying voltages with good 
regulation to avoid malfunction or undesired shutdown.

This work provides important advances with respect to [29], as for instance the experimental validation of the results, the certification of robustness to load variations and the investigation of viability of an $\mathscr{H}_{\infty}$ controller to deal directly with the control synthesis problem. In the sequence, this paper is organized as follows. In Section II, a model of the plant and a model of the augmented system are presented, including an integral action, as well as the requirements to be fulfilled by the closed-loop system. In Section III, a procedure for controller design is presented. Section IV includes a case study, with focus on an electrical vehicle, also showing the circuit used for analog control, information on the plant prototype and control implementation, a detailed procedure to compute the control gains and a study of viability of an $\mathscr{H}_{\infty}$ state feedback controller for this application. Section $\mathrm{V}$ describes the experimental tests, with analysis of the quality of the results, showing the practical viability of the control technique described in the paper. Section VI presents the main conclusions of this work.

\section{MODELLING}

Consider the circuit of a DC-DC buck converter [2], with disturbance on the input voltage, given in Figure 1.

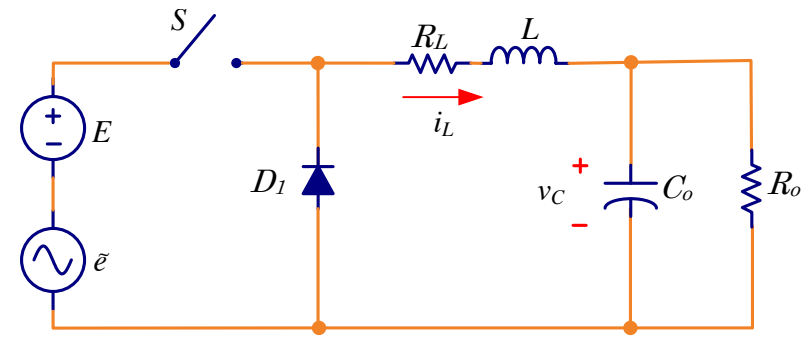

Fig. 1. Buck converter topology.

This converter can be used, for instance, to regulate the voltage that supplies auxiliary loads embedded in small size electrical vehicles. The behaviour of the battery is modelled by a fixed voltage source $E$, associated with a disturbance $\tilde{e}$. The losses on the inductor are modeled by $R_{L}$ and the load is described by the resistance $R_{o}$.

Consider that the converter is on the continuous conduction mode (CCM). A model can be obtained from the description of each of two operation mode in the state space. The description for the operation mode given by the switch $(S)$ in conduction, and the diode $\left(D_{1}\right)$ blocked, is defined by

$$
\dot{x}=A_{1} x+B_{1}(E+\tilde{e})
$$

where the dinamic matrix $A_{1}$, the input vector $B_{1}$ and the state vector $x$ are given by

$$
A_{1}=\left[\begin{array}{rr}
-\frac{R_{L}}{L} & -\frac{1}{L} \\
\frac{1}{C_{o}} & -\frac{1}{R_{o} C_{o}}
\end{array}\right], B_{1}=\left[\begin{array}{c}
\frac{1}{L} \\
0
\end{array}\right] \text { and } x=\left[\begin{array}{c}
i_{L} \\
v_{C}
\end{array}\right]
$$

The operation mode defined by the switch blocked and the diode in conduction is given by:

$$
\dot{x}=A_{2} x+B_{2}(E+\tilde{e})
$$

where $A_{2}=A_{1}$ and $B_{2}=0_{2 \times 1}$.

The weighted model from each of the above operation modes is described by

$$
\left[\begin{array}{c}
\dot{i}_{L} \\
\dot{v}_{C}
\end{array}\right]=\left(d A_{1}+\bar{d} A_{2}\right)\left[\begin{array}{c}
i_{L} \\
v_{C}
\end{array}\right]+\left(d B_{1}+\bar{d} B_{2}\right)(E+\tilde{e})
$$

where $\bar{d}=(1-d)$.

Since $A_{1}=A_{2}$ and $B_{2}=0_{2 \times 1}$, one has that

$$
\left[\begin{array}{c}
\dot{i}_{L} \\
\dot{v}_{C}
\end{array}\right]=\left[\begin{array}{rr}
-\frac{R_{L}}{L} & -\frac{1}{L} \\
\frac{1}{C_{o}} & -\frac{1}{R_{o} C_{o}}
\end{array}\right]\left[\begin{array}{c}
i_{L} \\
v_{C}
\end{array}\right]+\left[\begin{array}{c}
\frac{1}{L} \\
0
\end{array}\right] d E+\left[\begin{array}{c}
\frac{1}{L} \\
0
\end{array}\right] d \tilde{e}
$$

Observing the output stage of the converter, given by Figure 2, one can rewrite (4) as

$$
\left[\begin{array}{c}
\dot{i}_{L} \\
\dot{v}_{C}
\end{array}\right]=\left[\begin{array}{rr}
-\frac{R_{L}}{L} & -\frac{1}{L} \\
\frac{1}{C_{o}} & -\frac{1}{R_{o} C_{o}}
\end{array}\right]\left[\begin{array}{l}
i_{L} \\
v_{C}
\end{array}\right]+\left[\begin{array}{c}
\frac{1}{L} \\
0
\end{array}\right] u+\left[\begin{array}{c}
\frac{1}{L} \\
0
\end{array}\right] \tilde{u} .
$$

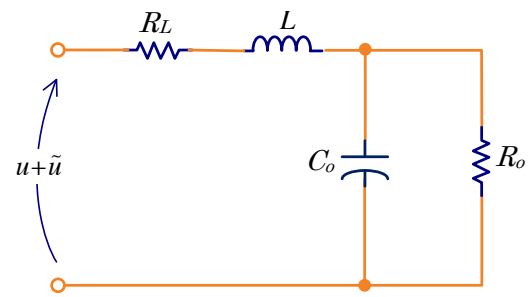

Fig. 2. Output stage of the converter.

Note that $d E$ can be replaced by the control signal $u$ and $d \tilde{e}$ can be replaced by the disturbance signal $\tilde{u}$, in the model of the plant given by (4). In practice, the control signal $u$ will be pulsewidth modulated (PWM) and $\tilde{u}$ will represent an exogenous disturbance on the input of the system. It is assumed also that the signal $u$ has limited bandwidth, so that it is suitably being representable in PWM. The high frequency components of $u$ in PWM will not be significant due to the low pass filter $(L C)$ on the output of the converter.

To ensure that the converter output voltage tracks a constant reference, with zero steady state error, it is necessary to include an integral action of the system error. The integral of the error can be included in the system model by means of an additional state variable $\xi$, as in [15], [30], leading to the representation

$$
\dot{\theta}=G \theta+H u+H_{d} \tilde{u}+H_{r} r
$$

where:

$$
\theta=\left[\begin{array}{c}
i_{L} \\
v_{C} \\
\xi
\end{array}\right], \quad G=\left[\begin{array}{cc}
A_{1} & 0_{2 \times 1} \\
J & 0
\end{array}\right]
$$




$$
\begin{gathered}
J=\left[\begin{array}{ll}
0 & -1
\end{array}\right], \\
H=\left[\begin{array}{c}
B_{1} \\
0
\end{array}\right], H_{d}=\left[\begin{array}{c}
B_{1} \\
0
\end{array}\right], H_{r}=\left[\begin{array}{c}
0_{2 \times 1} \\
1
\end{array}\right], \\
C=\left[\begin{array}{lll}
0 & 1 & 0
\end{array}\right] .
\end{gathered}
$$

In this description, $G$ is the dynamic matrix, $H$ is the control input vector, $H_{d}$ is the disturbance input vector, $H_{r}$ is the reference input vector, $C$ is the ouptut vector, $\theta$ is the augmented state vector and $r$ is the reference for output voltage.

The control law to be used is given by a state feedback, defined as

$$
u=-K \theta=-\left[\begin{array}{lll}
K_{1} & K_{2} & K_{3}
\end{array}\right]\left[\begin{array}{c}
i_{L} \\
v_{C} \\
\xi
\end{array}\right]
$$

with entries $K_{1}, K_{2}$ and $K_{3}$, to be computed in order to fulfill, simultaneously, the requirements:

(i) attenuation of disturbances on the input voltage, in the worst case, given by $\gamma$ (positive real). This value of $\gamma$ represents the peak of the magnitudes of the frequency responses from $\tilde{u}$ to $y$, as illustrated in Figure 3;

(ii) bound on the settling time for the transient responses depending on $\alpha$ (positive real), as illustrated in Figure 4, being $-\alpha$ the maximum real part of the closed-loop eigenvalues (dominant pole), which leads to the slowest mode in the transient responses with settling times bounded by $5 / \alpha$ (criterium of $1 \%$ of error) [31];

(iii) robustness against load resistance variations, in the real interval $R_{O} \in\left[R_{O_{\min }}, R_{O_{\max }}\right]$.

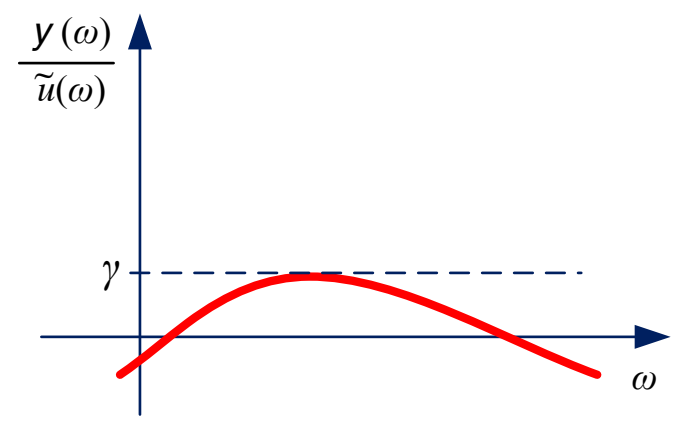

Fig. 3. Detail of the peak of the Bode magnitude plot $[y(\omega) / \tilde{u}(\omega)]$.

For instance, observe that lower values of $\gamma$ lead to better rejection of disturbances on the input voltage and that higher values of $\alpha$ lead to faster accommodation of the transient responses. Note that in general, the control designer has to establish a good trade-off between $\alpha$ and $\gamma$. Besides, the stability of the closed-loop system subject to arbitrary (slow or fast) variations of the load resistance, in the interval $\left[R_{O_{\min }}, R_{o_{\max }}\right]$, will be ensured by means of the existence of a Lyapunov function.

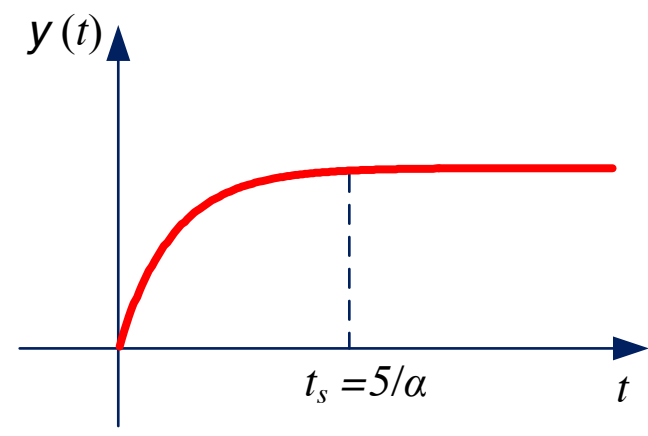

Fig. 4. Bound on the settling times for slowest mode in the transient responses, given by $5 / \alpha$.

\section{DESIGN OF THE CONTROLLER}

The design of the controller gains is based on the LQR, which aims on the minimization of the cost function [31]:

$$
\int_{0}^{\infty}\left(\theta^{\prime} Q \theta+u^{\prime} R u\right) d t
$$

where $\theta$ is the augmented state vector, $u$ is the control signal, the matrix $Q$ and the scalar $R$ come from heuristic choices of the control designer and, the superscritpt $\left({ }^{\prime}\right)$ represents the vector or matrix transposed.

In general, $Q$ is chosen as a positive diagonal matrix, where the diagonal entries give the relative importance of each state variable $\left(i_{L}, v_{C}\right.$ and $\xi$ ), and where the scalar $R$ allows to impose limits for the control action $(u)$. A higher value for an entry of the diagonal $Q$, generally leads to a faster and less oscillatory transient response for the state variable associated with this entry of $Q$. Increasing the value of $R$, one has a reduction of the control variable, allowing to avoid control saturation, for example.

The Riccatti equation [31]:

$$
G^{\prime} S+S G-S H R^{-1} H^{\prime} S+Q=0
$$

provides a solution for the matrix $S$, which leads to the gains of the LQR controller, given by

$$
K=R^{-1} H^{\prime} S
$$

which can be easily computed by the lqr function from MATLAB.

A procedure will be proposed here, to find the entries of $Q$ and $R$ that provides the gain vector $K$ suitable to fulfill the requirements (i), (ii) and (iii).

To verify if the requirement (i) was fulfilled, it is possible to use a test based on the $\mathscr{H}_{\infty}$ norm of the closed-loop system, which can be computed by means of the convex optimization problem based on LMIs [28]:

$$
\begin{gathered}
\mu^{*}=\min \mu \\
\text { s.t. } \\
P=P^{\prime}>0 \\
{\left[\begin{array}{cc}
(G-H K)^{\prime} P+P(G-H K)+C^{\prime} C & P H_{d} \\
H_{d}^{\prime} P & -\mu I
\end{array}\right]<0}
\end{gathered}
$$


where $G, H, H_{d}$ and $C$ are the matrices from the model of the augmented system given in (6), the gain $K$ is previously computed by (10), and the matrix $P$ and the scalar $\mu$ are the variables to be computed by the LMI solver.

In the case the $\mathscr{H}_{\infty}$ norm, given by $\sqrt{\mu^{*}}$, is less or equal to $\gamma$, the requirement $(i)$ is fulfilled. It is important to mention that, for a SISO system, the $\mathscr{H}_{\infty}$ norm is equal to the peak of the Bode magnitude plot from the input $\tilde{u}$ to the output $y$, as illustrated in Figure 3.

The requirement (ii) is fulfilled if

$$
\beta=\max \{\Re e(\lambda(G-H K))\}
$$

where $\beta$ is the maximum real part of the eigenvalues of the closed-loop system, and it is less or equal than $-\alpha$.

To ensure if the requirement (iii) is fulfilled, one verifies the existence of matrix $P$, solving the LMIs [28]:

$$
P=P^{\prime}>0:\left(G_{i}-H K\right)^{\prime} P+P\left(G_{i}-H K\right)<0, i=1,2 .
$$

Note that the LMIs in (13) must be solved simultaneously for two vertices $\left(G_{1}\right.$ and $\left.G_{2}\right)$, obtained from the evaluation of matrix $G$ for the minimum and maximum values of load resistance $R_{o}$. The gain vector $K=\left[\begin{array}{lll}K_{1} & K_{2} & K_{3}\end{array}\right]$ is previously computed by (9) and (10), which is the Riccatti's equation.

The existence of solution for (13) provides a quadratic Lyapunov function

$$
v(\theta)=\theta^{\prime} P \theta
$$

ensuring the stability of the closed-loop system for any variation of $R_{o}$ inside the interval $\left[R_{O_{\min }}, R_{o_{\max }}\right]$.

\section{CASE STUDY}

\section{A. Contextualization}

The design technique proposed here can be used in several contexts. One of them concerns electrical vehicle auxiliary sources [32], used to feed low voltage loads, as for instance, control systems (digital control, sensors and actuators) and lightning [33]. Usually, modern electrical vehicles employ a system with two sources: a main source, with higher voltage, for electrical propulsion of the vehicle, and an auxiliary source, with lower voltage, to feed low power loads [34].

The proposed controller can be used in a small size electrical vehicle, as the electrical kartcross, sketched in Figure 5. In this system, a buck converter is responsible to reduce the voltage level of the batteries to feed, with good regulation, loads at $5 \mathrm{~V}$, as for instance, digital signal controllers and analog circuits for signal conditioning.

It is interesting to observe that, usually, PI controllers are employed to get regulation for the output voltage of buck converters. However, in the usual methodologies for control design, robustness to parametric variations is not ensured theoretically for an entire domain of uncertainties with infinite points, but is only tested for some particular cases.

\section{B. Description of the Prototype}

To verify the efficiency of a controller obtained from the guidelines in Section III, consider the nominal parameters

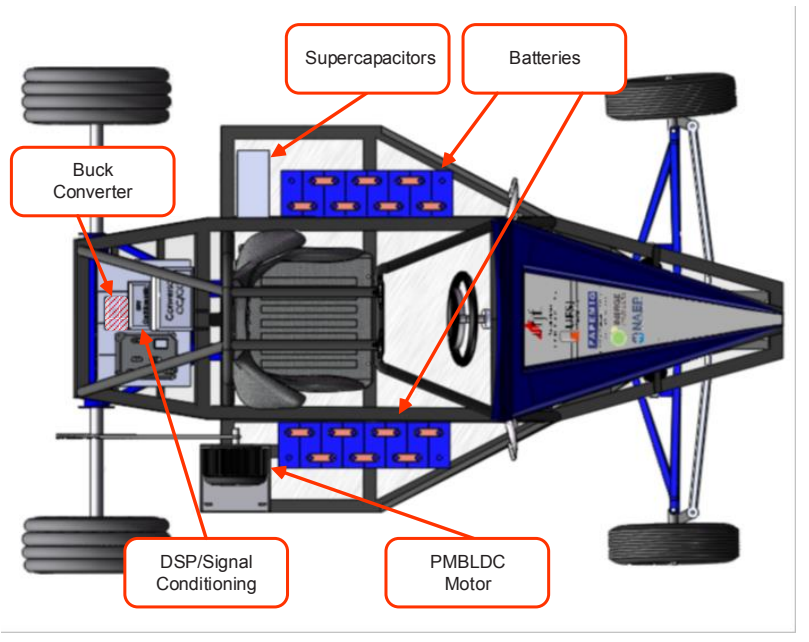

Fig. 5. Sketch of an electrical vehicle, detailing the buck converter for supplying auxiliary loads, described as DSP/Signal Conditioning.

given in Table I. It is assumed that the input voltage can vary in the interval from $20 \mathrm{~V}$ to $28.8 \mathrm{~V}$, the load resistance can vary in the interval from $1 \Omega$ to $3.5 \Omega$, the switching frequency is $20 \mathrm{kHz}$ and the output voltage must be regulated at $5 \mathrm{~V}$.

TABLE I

Nominal parameters of the buck converter

\begin{tabular}{c|c}
\hline Parameters & Values \\
\hline \hline$L$ & $1.2 \mathrm{mH}$ \\
\hline$R_{L}$ & $0.9 \Omega$ \\
\hline$C_{o}$ & $47 \mu \mathrm{F}$ \\
\hline$R_{o}$ & $1.5 \Omega$ \\
\hline
\end{tabular}

The practical implementation of the controller is carried out with analog circuits, using operational amplifiers as shown in Figure 6 . The gains of the controller were implemented by the choice of the resistors and capacitors, based on the expressions

$$
\left\{\begin{array}{l}
R_{2}=\frac{R_{f 1} R_{3}}{R_{1}} \\
R_{4}=\frac{R_{f 2}}{K_{2}} \\
R_{5}=\frac{R_{f 2}}{K_{1}} \\
R_{6}=\frac{R_{f 2}}{K_{3} C_{1} R_{3}} .
\end{array}\right.
$$

A photography of the built prototype in the laboratory is shown in Figure 7. The inductor current $\left(i_{L}\right)$ is measured by a current sensor ACS712-05B and the capacitor voltage $\left(v_{C}\right)$ is measured by a voltage sensor LV-25P.

\section{Example of Control Design}

The determination of the controller gains is carried out by means of a six step procedure described bellow:

Step 1: Compute the matrices of the system model by using (6) and the nominal parameters given in Table I; 


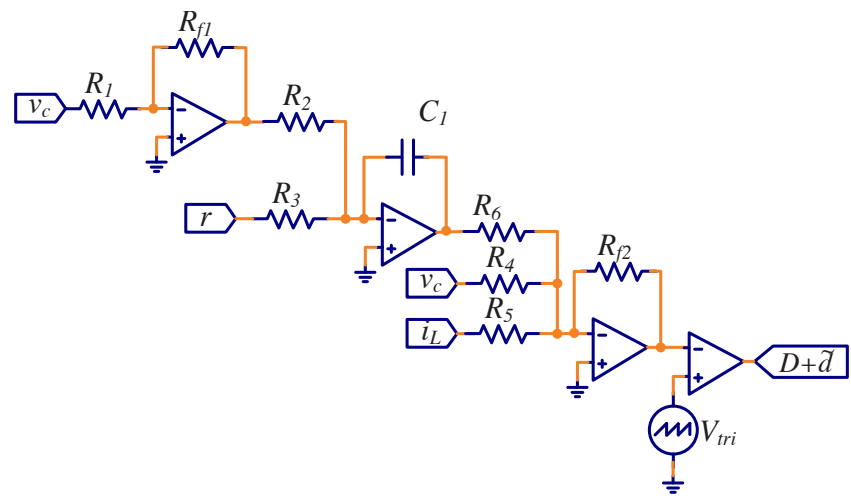

Fig. 6. Control circuit based on operational amplifiers.

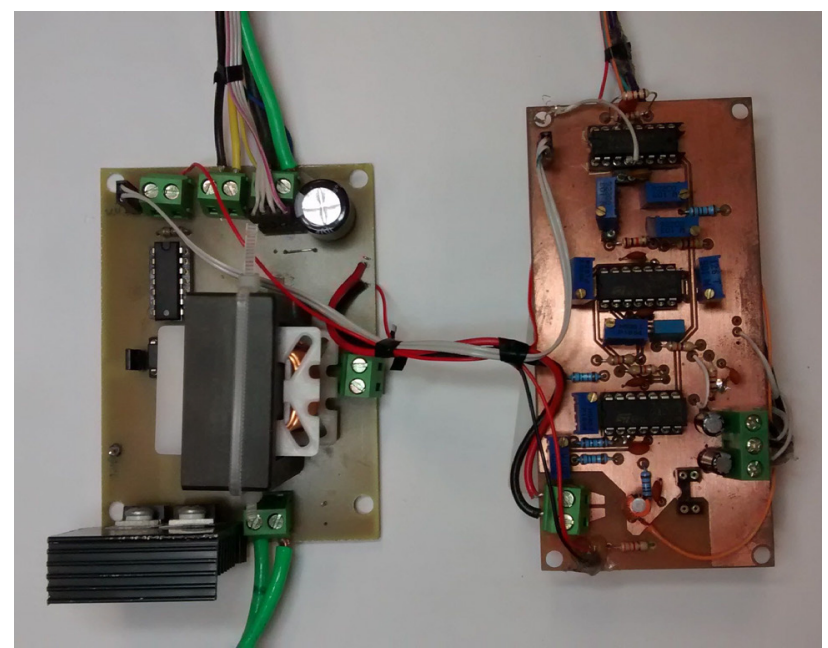

Fig. 7. Photograph of the prototype, with the buck converter (left) and control circuit (right).

Step 2: $\quad$ Inform $\alpha, \gamma$ and the limits of load resistance $R_{o_{\min }}$ and $R_{O_{\max }}$ (all positive real scalars);

Step 3: Choose the weighting matrices $Q$ and $R$ for the LQR controller;

Step 4: $\quad$ Solve the LQR problem using, for instance, the function lqr from MATLAB, which provides as solution the vector as follows $[\mathrm{K}, \mathrm{S}, \mathrm{e}]=$ $\operatorname{lqr}(\mathrm{G}, \mathrm{H}, \mathrm{Q}, \mathrm{R})$;

Step 5: $\quad$ Solve (11), (12) and (13) to compute $\mu^{*}, \beta$ and $P$, respectively;

Step 6: $\quad$ If $\sqrt{\mu^{*}} \leq \gamma$, if $\beta \leq-\alpha$ and if there exists $P$ solution of (13), then $K$ ensures that the requirements $(i)$ to (iii) are fulfilled and the control design is finished; otherwise, return to Step 3.

For attenuation of disturbances, the requirement $\gamma_{d B}=$ $-10 \mathrm{~dB}$ was used, which, converted into absolute value, results in $\gamma=1 / \sqrt{10}=0.31622$, as in Figure 3. For limitation of settling time of the slowest mode in the transient responses, it was chosen $\alpha=50$ as requirement, as in Figure 4 . The limits of load resistance were chosen as $R_{O_{\min }}=1 \Omega$ and $R_{o_{\max }}=3.5 \Omega$.

For the weighting matrix $Q$ of the $\mathrm{LQR}$, a diagonal positive matrix was chosen, with fixed values for the two first diagonal entries. The weight related with the third state variable, representing the integral of the error, will have higher relative importance here, and has its value to be searched in the positive integers. The weight of the control action, R, was defined as a value between 0 and 1, with a step of search of 0.001 , since this range resulted, in simulation tests, control gains that do not produce saturation of the control action, and also are viable for practical implementation with circuits based on operational amplifiers. Employing the procedure proposed in this section, with these initializations, one gets the weighting matrices:

$$
Q=\left[\begin{array}{ccc}
10 & 0 & 0 \\
0 & 10 & 0 \\
0 & 0 & 38600
\end{array}\right] \text { and } R=0.381
$$

that produce the gains:

$$
K^{\prime}=\left[\begin{array}{r}
6.440262137580129 \\
0.525278444645627 \\
-318.2959879703251
\end{array}\right]
$$

where the superscript $\left({ }^{\prime}\right)$ denotes the transpose.

For the implementation of the control circuit of Figure 6, the following values for resistors and capacitor can be used: $R_{1}=R_{3}=R_{f 1}=R_{f 2}=47 \mathrm{k} \Omega$ and $C_{1}=47 \mu \mathrm{F}$. The values of the other resistors are obtained from (15).

These control gains ensure the fulfillment of requirement (i), as can be verified by the frequency response of the closedloop system shown in Figure 8. The maximum value of the Bode magnitude plot is lower than $-10 \mathrm{~dB}$, as expected. This maximum can be also interpreted as the $\mathscr{H}_{\infty}$ norm of the closed-loop system, and is equal to $-16.1236 \mathrm{~dB}$ (or 0.15624, in absolute value) as indicated by the horizontal line in the Bode magnitude plot of Figure 8.

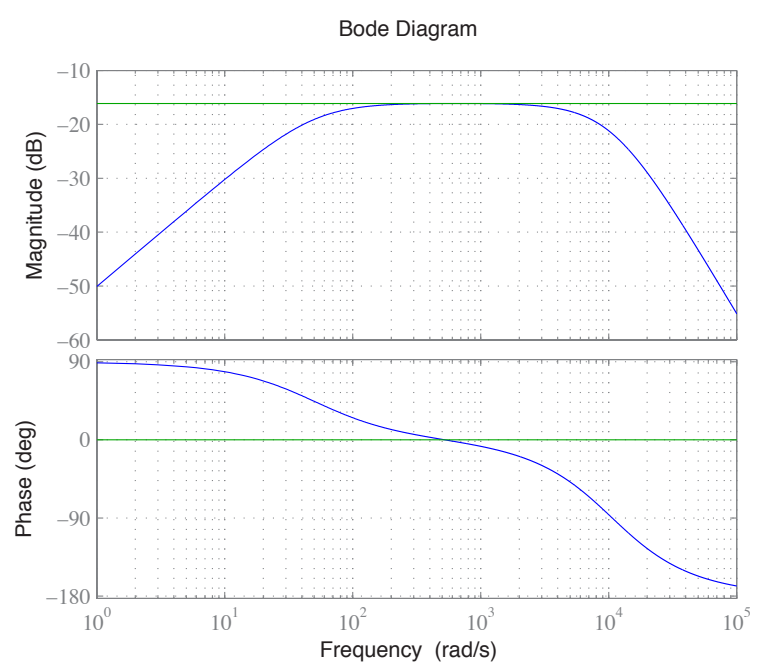

Fig. 8. Bode plots of $y / \tilde{u}$ for the closed-loop system with nominal parameters (maximum value of the magnitude curve $=-16.1236 \mathrm{~dB}$ ).

It is important to observe that the fulfillment of the predetermined attenuation for the disturbance $\tilde{u}$ ensures also the attenuation of the disturbance $\tilde{e}$. Observe that the duty cycle $d$ can be decomposed in $d=\tilde{d}+D$, being $D$ the value of the nominal duty cycle and $\tilde{d}$, in general, is given by a small disturbance on the nominal duty cycle. This allows to write 
$\tilde{u}=\tilde{d} \tilde{e}+D \tilde{e}$. In the case of small disturbances, $\tilde{d} \tilde{e}$ can be neglected and it is plausible the approximation $\tilde{u}=D \tilde{e}$. Thus,

$$
\frac{y}{\tilde{u}}=\frac{y}{D \tilde{e}} \rightarrow \frac{y}{\tilde{e}}=D \frac{y}{\tilde{u}} .
$$

As the duty cycle $0<D<1$, the peak of the Bode magnitude plot from $\tilde{e}$ to $y$ is always smaller than the peak of the Bode magnitude plot from $\tilde{u}$ to $y$, since

$$
\frac{y}{\tilde{e}}(d B)=20 \log _{10} D+\frac{y}{\tilde{u}}(d B) .
$$

The fulfillment of requirement (ii) can be verified by means of the closed-loop eigenvalues, given by

$\lambda=\left[\begin{array}{c}-1.012562431866448+j 0.320417858207570 \\ -1.012562431866448-j 0.320417858207570 \\ -0.005003364044169\end{array}\right] \times 10^{4}$

so that one can conclude that $\max \{\Re e(\lambda)\}<-50$ is verified.

The fulfillment of requirement (iii) is verified by the existence of the Lyapunov matrix, solution of (13), as shown below

$$
P=\left[\begin{array}{lll}
P_{1} & P_{2} & P_{3}
\end{array}\right]
$$

where $P_{1}, P_{2}$ and $P_{3}$ are vectors given by:

$$
\begin{gathered}
P_{1}=\left[\begin{array}{r}
0.0013686092642 \\
0.0001750309578 \\
-0.0043928720088
\end{array}\right] \\
P_{2}=\left[\begin{array}{r}
0.0001750309578 \\
0.0004207778385 \\
-0.0156445001309
\end{array}\right] \\
P_{3}=\left[\begin{array}{c}
-0.00439287200880 \\
-0.01564450013090 \\
234.6163318774911
\end{array}\right] .
\end{gathered}
$$

The matrix $P$ given in (21) ensures that the closed-loop system, with the gains computed by means of the proposed procedure, is quadratically stable as shown in [28]. In Figure 9 it is shown the locus of the eigenvalues for the compensated system considering $R_{O}$ varying from $1 \Omega$ to $100 \mathrm{k} \Omega$. The analysis of this figure shows that the closed-loop system remains stable even if for a great range of uncertainty of the load resistance.

\section{D. $\mathscr{H}_{\infty}$ Controller}

Finally, it is very important to observe that the design of a controller robust to uncertainties for the load in the interval $R_{o} \in\left[R_{o_{\text {min }}}, R_{O_{\text {max }}}\right]$, with maximum real part of the closed-loop eigenvalues given by $\alpha$, and with an $\mathscr{H}_{\infty}$ guaranteed cost given by $\gamma$ could be obtained directly from the condition of synthesis of a state feedback $\mathscr{H}_{\infty}$ controller based on quadratic stability [28].

The problem is defined as solving the following inequality by means of a LMI solver to determine a symmetric positive definite matrix $W=W^{\prime}>0$ and a matrix $Z$, such that,

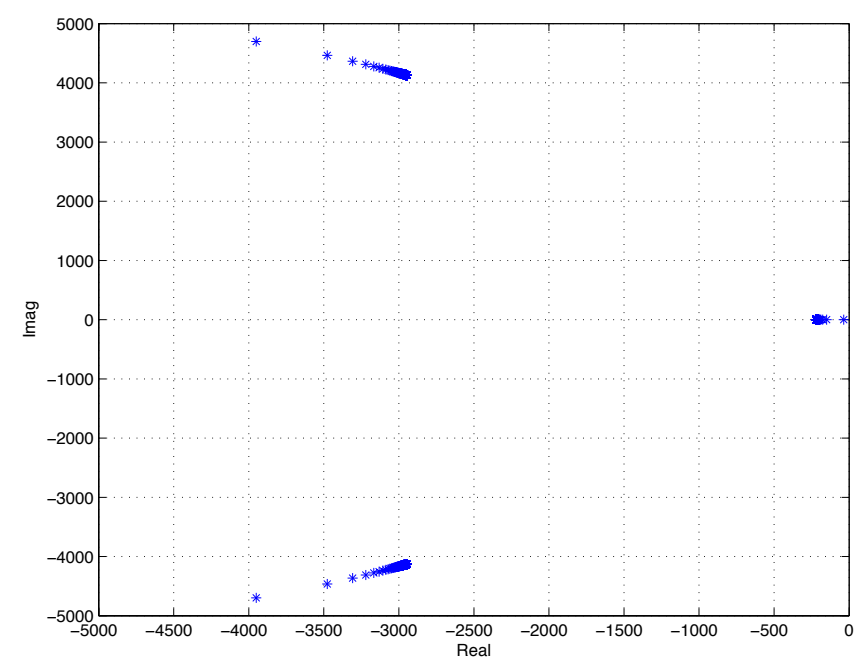

Fig. 9. Locus of the eigenvalues for the compensated system considering a large variation of the load $\left(1 \Omega \leq R_{o} \leq 100 \mathrm{k} \Omega\right)$.

$$
\left[\begin{array}{ccc}
\left(G_{i}+\alpha I\right) W+W\left(G_{i}+\alpha I\right)^{\prime}-H Z-Z^{\prime} H^{\prime} & W C^{\prime} & H_{d}^{\prime} \\
C W & -1 & 0 \\
H_{d}^{\prime} & 0 & \gamma^{2} I
\end{array}\right]<0
$$

for $i=1,2$.

If the above problem is feasible, the controller gains are obtained by means of the expression

$$
K=Z W^{-1} \text {. }
$$

Solving the above condition for $\gamma=0.31622(-10 \mathrm{~dB}), \alpha=$ 50 , and $R_{o} \in[1,3.5] \Omega$, the LMIs lead to the control gains

$$
K^{\prime}=\left[\begin{array}{r}
0.000001559471142 \\
0.046947661053007 \\
-7.983234084035900
\end{array}\right] \times 10^{7} .
$$

It is possible to conclude that the design of this state feedback $\mathscr{H}_{\infty}$ controller is less complex than the design of the state feedback controller proposed here, which is based on heuristic choices of $Q$ and $R$. However, the gains of the $\mathscr{H}_{\infty}$ controller, given in (24), are much higher than the gains in (17), obtained from the design procedure proposed here, so that (24) is not viable for practical implementation. Thus, a clear advantage in favor of the proposed controller (based on LQR) is its use in practical applications.

\section{EXPERIMENTAL RESULTS}

To verify the practical viability of the proposed controller, experimental results are presented in this section, illustrating the robustness to disturbances on the input voltage and stability to load variations.

Figure 10 presents the results for a negative and, in the sequence, a positive disturbance on the input voltage. The system initiates its operation with an input voltage of $28.8 \mathrm{~V}$. Then, a step on the input voltage is applied, varying its value to $20 \mathrm{~V}$. After that, a positive step is applied, driving the input voltage to its initial value. For application of these disturbances on the input voltage, in practice, an adjustable 
DC source was used.

Figure 11 presents the results to variations on the load resistance. Initially, the converter operates with a resistive load demanding $1.5 \mathrm{~A}$ at $5 \mathrm{~V}$, when a sudden variation of the resistive load is applied, demanding $3.0 \mathrm{~A}$ at $5 \mathrm{~V}$. After that, the resistive load returns to its initial value. One can observe a good regulation of the output voltage, with settling time lower than $5 / 50 \mathrm{~s}$.

It is worth mentioning that the result shown in Figure 11 was obtained considering a switched load which is, in this application, a simple example of a nonlinear load. It was not the objective of this work the investigation of the performance of the controller when the converter feeds other types of nonlinear loads. However, a good strategy to provide the attenuation of harmonics generated by nonlinear loads could be used, for example, following the ideas presented in [15].

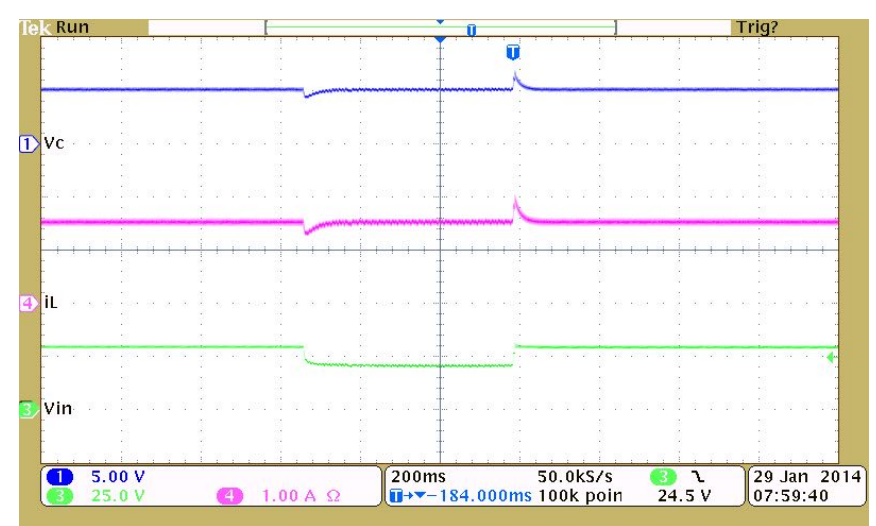

Fig. 10. Results for disturbances on the input voltage. Output voltage (top), inductor current (middle), and input voltage (bottom) waveforms.

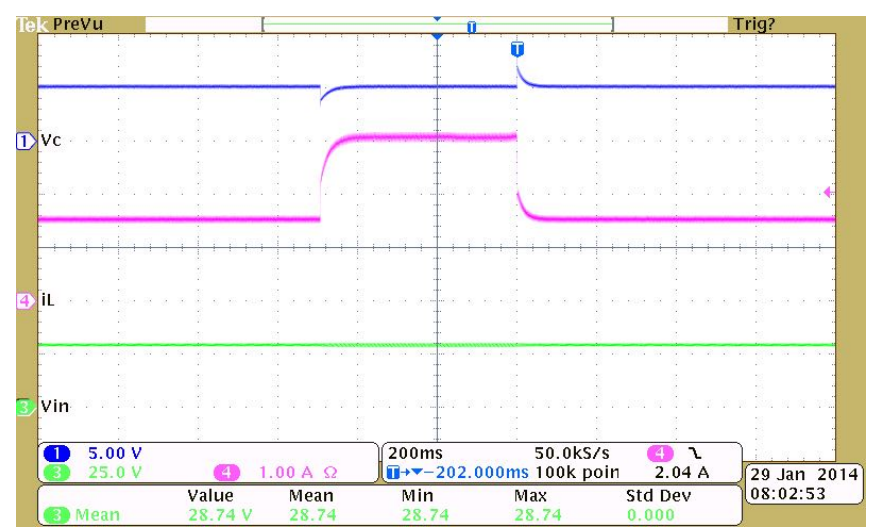

Fig. 11. Results for variations on the load resistance. Output voltage (top), inductor current (middle), and input voltage (bottom) waveforms.

The experimental result indicates a good performance, showing rapid recover at the transients and also zero steady state error. It is also possible to observe that controller assures the closed-loop stability even under sudden load variations.

\section{CONCLUSION}

This paper presented a procedure to design the gains of a state feedback controller that ensures the rejection of disturbances on the input voltage, a predetermined bound for the settling time of the slowest mode in the transient responses and robustness of the closed-loop system to variations on the load resistance. The gains of the controller are obtained from a LQR, and are certified using the $\mathscr{H}_{\infty}$ norm, the analysis of the closed-loop eigenvalues and the existence of a Lyapunov function for the quadratic stability of the system. A case study is detailed, emphasizing a practical application for the proposed controller in the context of an electrical vehicle. The design of the controller gains is described in a step by step procedure. Additionally, information on the prototype and on the practical implementation of the circuit of control are presented. Finally, experimental results illustrate the viability of the proposed design procedure, with responses of good quality in terms of rejection of disturbances, fast transient and robustness to load variations.

Nevertheless, comparing to classic control techniques, the great advantage of the proposed method is not necessarily a better performance but a control design procedure whose the solution is computationally fastest and which ensures the properties highlighted in the paper: $(i)$ a bound on the rejection of disturbances, certified by an $\mathscr{H}_{\infty}$ analysis; (ii) a bound on the settling times of the transient responses, ensured by an eigenvalue evaluation; (iii) the robustness of the closedloop system under load variations, ensured by a Lyapunov condition.

\section{ACKNOWLEDGMENT}

The authors would like to register their gratitude to the following development agencies to the partial financial support for this work: CAPES, CNPq, FAPEMIG and INCT/INERGE (National Institute of Science and Technology for Electric Energy).

\section{REFERENCES}

[1] S. Buso, P. Mattavelli, Digital Control in Power Electronics, Morgan \& Claypool Publishers, 2006.

[2] R. W. Erickson, D. Maksimovic, Fundamentals of power electronics, Springer Science \& Business Media, 2007.

[3] C.-T. Chen, Linear system theory and design, Oxford University Press, Inc., 1995.

[4] P. Dorato, V. Cerone, C. Abdallah, Linear-quadratic control: an introduction, Simon \& Schuster, 1994.

[5] F. H. Leung, P. K. Tam, C. Li, "An improved LQRbased controller for switching DC-DC converters", IEEE Transactions on Industrial Electronics, vol. 40, no. 5, pp. 521-528, Oct. 1993.

[6] C. O. Moreira, F. Silva, S. F. Pinto, M. B. Santos, et al., "Digital LQR control with Kalman Estimator for DC-DC Buck converter", in 2011 IEEE EUROCONInternational Conference on Computer as a Tool (EUROCON), pp. 1-4, 2011.

[7] V. F. Montagner, E. G. Carati, H. Grundling, et al., "Design and analysis of a linear quadratic regulator with repetitive controller for ac power supplies", in 2003 IEEE International Symposium on Industrial Electronics(ISIE'03), vol. 1, pp. 544-549, 2003. 
[8] R. Priewasser, M. Agostinelli, C. Unterrieder, S. Marsili, M. Huemer, "Modeling, control, and implementation of DC-DC converters for variable frequency operation", IEEE Transactions on Power Electronics, vol. 29, no. 1, pp. 287-301, Jan. 2014.

[9] F. Dupont, V. Montagner, J. Pinheiro, H. Pinheiro, S. Oliveira, A. Péres, "Comparison of Linear Quadratic Controllers with Stability Analysis for DC-DC Boost Converters Under Large Load Range", Asian Journal of Control, vol. 15, no. 3, pp. 861-871, May 2013.

[10] M. Mahdavian, M. B. Poudeh, S. Eshtehardiha, "DC-DC converter with closed loop control through several optimizing methods", in 11th International Conference on Optimization of Electrical and Electronic Equipment, pp. 233-238, 2008.

[11] H. Sira-Ramirez, M. A. Oliver-Salazar, "On the robust control of Buck-converter DC-motor combinations", IEEE Transactions on Power Electronics, vol. 28, no. 8, pp. 3912-3922, Aug. 2013.

[12] E. W. Zurita-Bustamante, J. Linares-Flores, E. Guzmán-Ramírez, H. Sira-Ramírez, "A comparison between the GPI and PID controllers for the stabilization of a DC-DC Buck converter: a field programmable gate array implementation", IEEE Transactions on Industrial Electronics, vol. 58, no. 11, pp. 5251-5262, Nov. 2011.

[13] R. de Castro, R. E. Araujo, J. P. F. Trovão, P. G. Pereirinha, P. Melo, D. Freitas, "Robust DC-link control in EVs with multiple energy storage systems", IEEE Transactions on Vehicular Technology, vol. 61, no. 8, pp. 3553-3565, Oct. 2012.

[14] C. Olalla, R. Leyva, A. El Aroudi, I. Queinnec, "Robust LQR control for PWM converters: an LMI approach", IEEE Transactions on Industrial Electronics, vol. 56, no. 7, pp. 2548-2558, July 2009.

[15] L. A. Maccari, V. F. Montagner, H. Pinheiro, R. C. Oliveira, "Robust $H_{2}$ control applied to boost converters: design, experimental validation and performance analysis", IET Control Theory \& Applications, vol. 6, no. 12, pp. 1881-1888, Aug. 2012.

[16] C. Olalla, R. Leyva, A. El Aroudi, P. Garces, I. Queinnec, "LMI robust control design for boost PWM converters", IET Power Electronics, vol. 3, no. 1, pp. 75-85, Jan. 2010.

[17] C. Olalla, I. Queinnec, R. Leyva, A. El Aroudi, "Robust optimal control of bilinear DC-DC converters", Control Engineering Practice, vol. 19, no. 7, pp. 688-699, July 2011.

[18] G. S. Deaecto, J. C. Geromel, F. Garcia, J. Pomilio, "Switched affine systems control design with application to DC-DC converters", IET control theory \& applications, vol. 4, no. 7, pp. 1201-1210, July 2010.

[19] T. Hu, "A nonlinear-system approach to analysis and design of power-electronic converters with saturation and bilinear terms", IEEE Transactions on Power Electronics, vol. 26, no. 2, pp. 399-410, Feb. 2011.
[20] L. Martínez-Salamero, G. García, M. Orellana, C. Lahore, B. Estibals, C. Alonso, C. E. Carrejo, "Analysis and design of a sliding-mode strategy for start-up control and voltage regulation in a buck converter", IET Power Electronics, vol. 6, no. 1, pp. 52-59, Jan. 2013.

[21] H. de Battista, R. J. Mantz, "Variable structure control of a photovoltaic energy converter", IEE ProceedingsControl Theory and Applications, vol. 149, no. 4, pp. 303-310, July 2002.

[22] Y. He, F. Luo, "Sliding-mode control for dc-dc converters with constant switching frequency", IEE Proceedings - Control Theory and Applications, vol. 153, no. 1, pp. 37-45, Jan. 2006.

[23] A. Fradkov, J. Alvarez, D. Cortes, "Tracking control of the boost converter", IEE Proceedings - Control theory and applications, vol. 151, no. 2, pp. 218-224, March 2004.

[24] R. Leyva, A. Cid-Pastor, C. Alonso, I. Queinnec, S. Tarbouriech, L. Martinez-Salamero, "Passivitybased integral control of a boost converter for largesignal stability", IEE Proceedings - Control Theory and Applications, vol. 153, no. 2, pp. 139-146, March 2006.

[25] H. Lam, S. Tan, "Stability analysis of fuzzy-modelbased control systems: application on regulation of switching DC-DC converter", IET control theory \& applications, vol. 3, no. 8, pp. 1093-1106, Aug. 2009.

[26] S.-K. Kim, J.-S. Kim, C. R. Park, Y. I. Lee, "Outputfeedback model predictive controller for voltage regulation of a DC/DC converter", IET Control Theory \& Applications, vol. 7, no. 16, pp. 1959-1968, Nov. 2013.

[27] K. Zhou, J. C. Doyle, K. Glover, et al., Robust and optimal control, vol. 40, Prentice Hall, 1996.

[28] S. P. Boyd, V. Balakrishnan, Linear matrix inequalities in system and control theory, vol. 15, SIAM - Studies in Applied Mathematics, 1994.

[29] L. A. Maccari, V. Foletto Montagner, A. Ferreira, et al., "A linear quadratic control applied to buck converters with H-infinity constraints", in Power Electronics Conference (COBEP), 2013 Brazilian, pp. 339-344, 2013.

[30] B. A. Francis, W. M. Wonham, "The internal model principle of control theory", Automatica, vol. 12, no. 5, pp. 457-465, Sep. 1976.

[31] K. Ogata, Modern control engineering, Prentice-Hall, 1990.

[32] D. Gosden, "Electric vehicle auxiliary power supply", in Proceedings of the Institution of Electrical Engineers, vol. 123, pp. 711-712, 1976.

[33] A. Emadi, Y. J. Lee, K. Rajashekara, "Power electronics and motor drives in electric, hybrid electric, and plug-in hybrid electric vehicles", IEEE Transactions on Industrial Electronics, vol. 55, no. 6, pp. 2237-2245, June 2008.

[34] A. Emadi, S. S. Williamson, A. Khaligh, "Power electronics intensive solutions for advanced electric, hybrid electric, and fuel cell vehicular power systems", 
IEEE Transactions on Power Electronics, vol. 21, no. 3, pp. 567-577, May 2006.

\section{BIOGRAPHIES}

Luiz Antonio Maccari Junior received the bachelor, master and doctor degrees in Electrical Engineering from Federal University of Santa Maria in 2009, 2011 and 2015 respectively. Since 2014 he is Assistant Professor at the Universidade Federal do Pampa (UNIPAMPA), RS, Brasil.

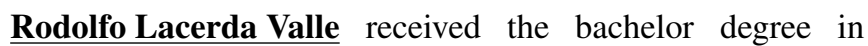
Control and Automation Engineering from CEFET-MG, in 2010 and the master degree in Electrical Engineering from Federal University of Juiz de Fora in 2013, where he is pursing his doctorate. Since 2014 he is EBTT professor at Centro Federal de Educação Tecnológica de Minas Gerais (CEFETMG), Leopoldina, MG, Brasil.

André Augusto Ferreira received the bachelor degree in Electrical Engineering from Federal University of Juiz de Fora in 2000 and the master and doctor degrees, both in Electrical Engineering, from State University of Campinas,
SP, in 2002 and 2007, respectively. Currently, he is professor at the Federal University of Juiz de Fora, Juiz de Fora, Brazil. His research interest includes dc-dc power converters control, digital control and power quality.

Pedro Gomes Barbosa received the bachelor degree in Electrical Engineering from Federal University of Juiz de Fora in 1986 and the master and doctor degrees, both in Electrical Engineering, from Federal University of Rio de Janeiro, in 1994 e 2000, respectively. Currently, he is professor at the Federal University of Juiz de Fora, Juiz de Fora, Brazil. His main research interests are modeling and control of power electronics converters, integration of renewable energy sources and active power filters.

Vinícius Foletto Montagner received the bachelor and the master degrees in Electrical Engineering, in 1996 and 2000, from Federal University of Santa Maria and the doctor degree in Electrical Engineering from State University of Campinas in 2005. Currently, he is professor at Electric Power Processing Department in Federal University of Santa Maria. His research interests include control theory and applications. 